\title{
The Disobeying 'Soldier': Use of an Achiral Group to Modulate Chiral Induction in PNA Duplexes
}

\author{
Artur Sargun, Yanhaomin Fang, and Catalina Achim*
}

\begin{abstract}
Peptide nucleic acid (PNA) is a synthetic analogue of DNA in which the natural nucleobases A, G, C, and $T$ are linked to an achiral, charge neutral, pseudopeptide backbone. PNA strands can form double helices similar to DNA whose helical sense can be modulated by applying the 'sergeants-and-soldiers' principle. Attachment of a chiral amino acid (sergeant) at the C-terminus of PNA leads to the amplification of chirality of the sergeant onto the achiral PNA monomers (soldiers), resulting in an enantiomeric excess of either left- or right-handed PNA duplexes. In the present study we looked at the effect of an achiral N-terminal terpyridine (soldier) on the helicity of the double helix that contains L-lysine. We have found that terpyridine interferes with the chiral induction effect of the L-lysines, an effect that can be reverted upon coordination of $\mathrm{Cu}^{2+}$ ions to terpyridine.
\end{abstract}

Keywords: Coordination complexes · Inversion of handedness · Peptide nucleic acids · Sergeants and soldiers effect $\cdot$ Terpyridine
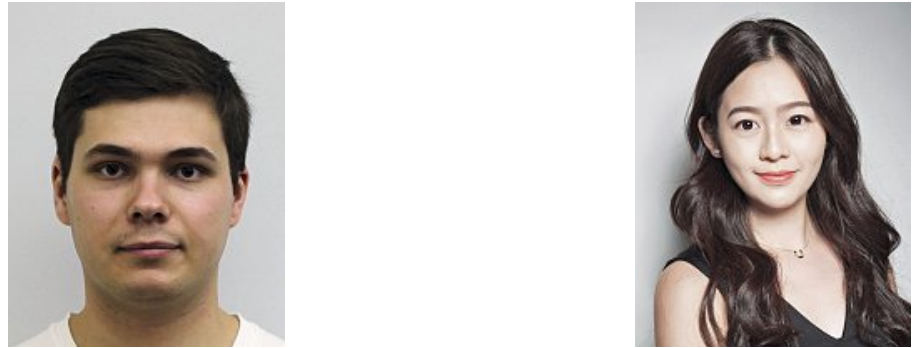

Yanhaomin Fang is an undergraduate

Artur Sargun is a $\mathrm{PhD}$ candidate in the laboratory of Professor Catalina Achim at Carnegie Mellon University, the USA. He received his BSc and MSc in Chemistry from Moldova State University where he worked on heterometallic coordination complexes as precursors to mixed-oxide systems as well as on the design of molecules with anticancer and antibacterial properties. Currently, Artur studies metalmodified peptide nucleic acids and their applications in supramolecular chemistry and nanotechnology.
${ }^{\star}$ Correspondence: Prof. C. Achim

Carnegie Mellon University

Department of Chemistry

4400 Fifth Avenue, Pittsburgh PA 15213, USA

E-mail: achim@cmu.edu student in the Department of Chemistry at Carnegie Mellon University. Yanhaomin does research in the laboratory of Professor Catalina Achim where she studies the methods of induction of handedness in ligand-containing peptide nucleic acid duplexes as well as the impact of transition metal ion coordination upon the chiroptical properties of the assembled helical systems.

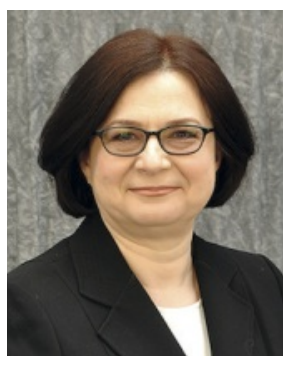

Catalina Achim is a Professor in the Department of Chemistry at Carnegie Mellon University, in Pittsburgh, the USA. She holds a BS/MS degree from the Polytechnical Institute of Bucharest in
Romania, and a PhD from Carnegie Melon University, both in Chemistry. Between 1999-2001, she was a postdoctoral fellow at Harvard University. Then she returned to her alma mater to serve as Professor in the Chemistry Department at Carnegie Mellon. Her research interests include inorganic supramolecular chemistry with a focus on the synthesis of metal-containing, nucleic acid-based nanostructures, and physical inorganic chemistry with a focus on polynuclear complexes with spin transitions.

\section{Introduction}

The design and study of supramolecular helical systems is a topic of great interest due to the importance of chiroptical properties in the production of sensors, ${ }^{[1]}$ optoelectronic devices, ${ }^{[2]}$ photochromic materials, ${ }^{[3]}$ as well as in the emerging field of spintronics. ${ }^{[4]}$ Many artificial helices have been designed to mimic the natural helical macromolecules (e.g. DNA and proteins) in order to take advantage of such powerful tools of self-assembly as $\mathrm{H}$-bonding, $\pi$-stacking, and metal-ligand coordination. ${ }^{[5]}$

The preference for one helical sense in the self-assembled synthetic structures can be induced by applying the "sergeants-andsoldiers' principle, according to which one chiral unit (a sergeant) relays its handedness to the rest of the structure made of achiral moieties (soldiers) by means of noncovalent interactions. ${ }^{[6]}$ To date, numerous chiral systems have been successfully assembled using this principle. ${ }^{[7]}$ 
Since recent research has shown that chiral self-assembled nanostructures can act as spin filters, ${ }^{[8]}$ the possibility of inverting the helical sense of an already assembled system could open the path towards nanodevices with switchable and tunable physicochemical properties.

Here we discuss means to affecting and inverting the handedness of a nucleic acidbased duplex by meddling in the communication between the chiral 'sergeant' and the achiral 'soldiers'. The unique double helix structure of DNA inspired the biomimetic synthesis of numerous helices. ${ }^{[9]}$ However, due to the inherent chirality of deoxyribose, there are only limited possibilities for inversion of handedness in DNA duplexes, such as a change from right-handed $\mathrm{B}$ to left-handed $\mathrm{Z}$ double helix by changing the ionic strength of the solution. ${ }^{[10]}$ If the backbone of the nucleic acid would be achiral, a double-stranded structure formed by Watson-Crick hybridization from complementary strands could still be helical. However, the incorporation of one or several chiral moieties in a system devoid of intrinsic chiral centers could induce a preferred handedness through the 'sergeants-and-soldiers' effect. A DNAlike system that meets this description is Peptide Nucleic Acid.

Peptide Nucleic Acids (PNAs) are synthetic analogs of DNA that typically have an electroneutral and achiral $N-(2-$ aminethyl)glycine-based backbone. In PNA, the four natural nucleobases A, G, $\mathrm{C}$, and $\mathrm{T}$ are connected to the pseudopeptide backbone via a methylene carbonyl linker, and are able to form Watson-Crick base pairs similar to those of DNA (Fig. 1). As a result, PNA strands can hybridize to complementary DNA, RNA, or PNA forming double helices. ${ }^{[11]}$

PNA duplexes adopt in solution and crystals a P-form helical structure with a large pitch that has $\sim 18$ base pairs per turn and a diameter of $28 \AA .{ }^{[12]}$ Since the PNA backbone is achiral, an equal mixture of right- and left-handed helices is present in solutions of PNA duplexes; no $\mathrm{CD}$ is observed for such solutions. From the earliest reports on PNA in the 1990s, it was noted that an L-lysine attached at the carboxamide terminus (C-terminus) of PNA induces in the PNA duplexes a preference for left-handedness. This is reflected in the observation of a characteristic CD spectrum with negative peaks at 220 and $256 \mathrm{~nm}$, and a small positive peak at 278 nm. ${ }^{[11]}$ Since 1990 s, a preferred handedness has been induced in PNA duplexes by use of either chiral amino acids or of PNA monomers that have a chiral center in their backbone. The chiral induction effect was manifested irrespective of where the chiral group was placed, i.e. at the end or in the middle of the duplex's strands.

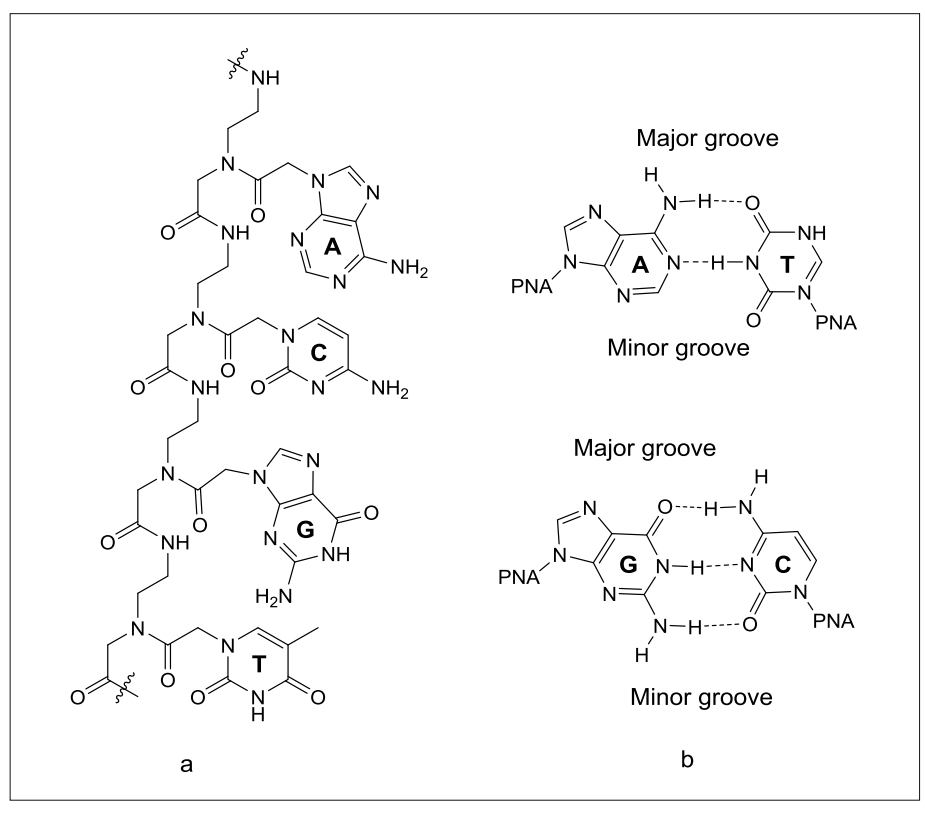

Fig. 1. (a) Chemical structure of PNA; (b) Watson-Crick nucleobase pairing.

Nielsen and coworkers investigated the influence of various amino acids on the helical sense of PNA duplexes and concluded that L-amino acids with hydrophobic side chains tend to generate left-handed duplexes, whereas amino acids with hydrophilic side chains - right-handed duplexes. ${ }^{[13]}$ In the same study, it was found that the chiral influence of terminal amino acids extends up to the tenth base pair, suggesting this number as a limit for the influence of the terminal amino acid on the helical sense of the PNA duplex. ${ }^{[13]}$ Subsequent studies by Green and collaborators led to the conclusions that (1) the PNA duplexes exist in solution as a dynamic ensemble of conformational states whose distribution depends on the nature of the terminal amino acid and on the sequence and length of the duplexes, (2) the terminal amino acid exerts not only a chiral effect, but a general structural effect on the duplex that extends beyond the nucleobases proximal to the amino acid, and (3) the limit of the chiral induction effect of the terminal amino acid may be longer than 10 base pairs. ${ }^{[14]}$ These experimental data are compatible with a structural model in which PNA duplexes are intrinsically conformationally heterogeneous. Attachment of a chiral amino acid may restrict the manifold of conformers to a smaller set, but the system remains multistate in nature. ${ }^{[14]}$ Computational studies carried out by Rasmussen et al. on a selfcomplementary PNA hexamer have shown that the preference for the left-handed helix in a duplex with L-lysine may be due to cation- $\pi$-interaction between the $\mathrm{NH}_{3}{ }^{+}$of the L-lysine and the guanine ring system, as well as to differences in the free energies of hydration. ${ }^{[15]}$

In contrast to solution, a unique helical conformation is observed by X-ray crystallography in PNA crystals. ${ }^{[16]}$ Our previous studies of the structure of a pal- indromic 8-base pair PNA duplex containing C-terminal L-lysines identified the presence in the crystal of a racemic mixture of right- and left-handed duplexes. ${ }^{[17]}$ Moreover, we have identified H-bonds between, on one side, the backbone and the side-chain of the C-terminal L-lysine of the duplex and the backbone or nucleobases close to the end of neighboring PNA duplexes on the other side (Fig. 2). ${ }^{[17]}$ These interactions may manifest also in solution and underlie the chiral induction effect of the lysine on the duplex.

Alternative to the induction of a preferred handedness by a terminal chiral moiety, a preferred handedness can also be induced in PNA duplexes by one or more PNA monomers that contain a stereogenic center at the $\gamma$-position of the $N$-(2aminoethyl)glycine backbone[18] (Fig. 3).

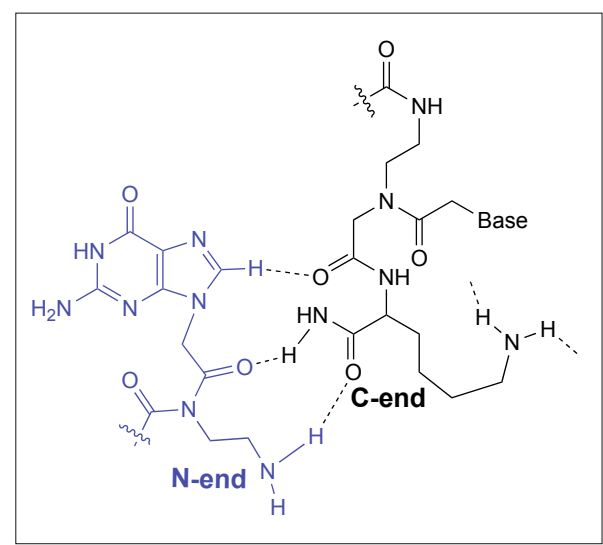

Fig. 2. The H-bonding sites of a C-terminal L-lysine in an 8-base pair PNA duplex are identified by dashed lines. ${ }^{[17]}$ The atoms of the $G$ PNA monomer situated on a complementary strand (blue) that participate in the $\mathrm{H}$-bonding are also identified. The $\mathrm{H}$ atoms of the amino group on the side arm of the L-lysine also participate in $\mathrm{H}$-bonding involving the $\mathrm{H}$-acceptor amide sites of another duplex (not shown). 
Fig. 3. (a) Achiral $N$-(2-aminoethyl) glycine-based PNA monomer; (b) Chiral (S) $\gamma$-methyl PNA monomer.

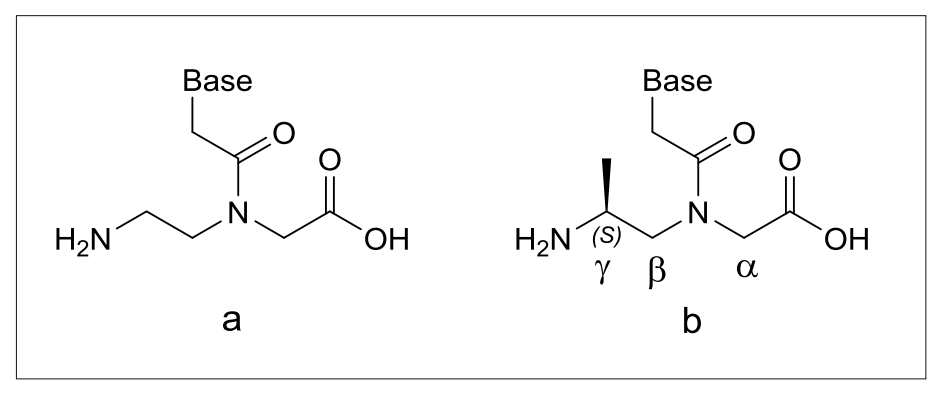

For example, an (S)-methyl stereogenic center at the $\gamma$-position in the backbone induces the preference for a right-handed helix in both single-stranded and duplex PNA. [18]

We built on the knowledge of chiral induction effects of amino acids and chiral PNA monomers to achieve helical sense inversion in PNA via metal-ligand coordination. Two C-terminal chiral moieties (sergeants) were attached to the PNA strands; namely an L-lysine and a $(S) \gamma$-methyl Tpy PNA monomer ${ }^{[19]}$ (Fig. 4a). In the absence of a chiral $\gamma$-PNA monomer, the L-lysine would confer to the duplex a left-handed structure, whereas in the absence of L-lysine, the $(S) \gamma$-methyl Tpy PNA monomer would induce a right-handed structure. A duplex that contains both units experiences two conflicting helical sense induction effects. In the absence of transition metal ions, PNA duplexes that contained both 'sergeants' at opposite ends adopted a right-handed structure; the chiral PNA monomer was the 'winner'. Upon addition of $\mathrm{Cu}^{2+}$ ions, the handedness of the duplex reverted to left. This switch in handedness was attributed to the fact that the chiral induction effect of the $(S) \gamma$-methyl terpyridine PNA monomer, which was underscored by $\pi$-stacking of the terpyridine at the $\mathrm{C}$-end of the duplex, manifests in the absence but not in the presence of $\mathrm{Cu}^{2+}{ }^{[}{ }^{1}$ ]

In the present work, we hypothesized that if a chemical group capable of interacting with L-lysine is placed opposite to the terminal lysine in a PNA duplex, it can modulate the chiral induction effect of the amino acid. That chemical group would behave as a disobeying 'soldier' that interferes with the orders of the 'sergeant'. If the chemical group is a metal-binding ligand whose interaction with the lysine is turned on/off by metal coordination, it could act as a switch for the PNA duplex handedness. To test this hypothesis, we examined three PNA duplexes that contained an achiral 2,2':6',2"-terpyridine (Tpy) moiety (Fig. 4b) in a position complementary to the C-terminal L-lysine. Indeed, we observed that the achiral Tpy (soldier) interferes with the induction effect of the chiral L-lysine (sergeant). Then, the coordination ability of Tpy was exploited to modulate the handedness of the Tpy-containing duplex by means of $\mathrm{Cu}^{2+}$ coordination.

\section{Results}

The antiparallel PNA duplex $\mathrm{P}_{\mathrm{N}}$ shown in Table 1 had been investigated extensively since 1994.[11] In the present study, we have synthesized three double-stranded PNAs whose sequences are related to that of the $\mathrm{P}_{\mathrm{N}}$ duplex. The sequences and designation of the duplexes used throughout this work are given in Table 1. The duplexes ${ }^{G, l y s} \boldsymbol{T} \boldsymbol{p y} \mathrm{P}_{\mathrm{N}},{ }^{A, l y s} \boldsymbol{T} \boldsymbol{p y} \mathrm{P}_{\mathrm{N}}$, and ${ }^{G} \boldsymbol{T} \boldsymbol{p} \boldsymbol{y} \mathrm{P}_{\mathrm{N}}$ contain a $2,2^{\prime}: 6^{\prime}, 2^{\prime \prime}$-terpyridine attached to the terminal amine of a PNA strand using the $\left[2,2^{\prime}: 6^{\prime}, 2^{\prime \prime}\right.$-terpyridine]-4'-carboxylic acid (Fig. 4b). In duplexes ${ }^{G, l y s}$ Tpy $\mathrm{P}_{\mathrm{N}}$ and ${ }^{A, l y s}$ Tpy $\mathrm{P}_{\mathrm{N}}$ an L-lysine is attached at the C-terminus of the complementary strand. Hence, these two duplexes contain a terpyridine and L-lysine complementary to each other at the end of the duplex, and next to a G-C or an A-T base pair. Duplex ${ }^{G}$ Tpy $P_{\mathrm{N}}$ contains a terpyridine unit and an L-lysine, but they are at opposite ends of the same strand. The ${ }^{\gamma} \boldsymbol{T} \boldsymbol{p y} \mathrm{P}_{\mathrm{N}}$ duplex studied by Bezer et al. ${ }^{[19]}$ contained an $(S) \gamma$-methyl Tpy monomer attached at the $\mathrm{C}$-end of the PNA oligomer (Fig. 4a).

\subsection{Variable Temperature UV-Vis Spectroscopy}

The thermal stability of the synthesized PNA duplexes in the presence and absence of $\mathrm{Cu}^{2+}$ ions was studied by monitoring the absorbance at $\lambda_{\max }=260 \mathrm{~nm}$ of the nucleobases as function of temperature. The thermal annealing curves are shown PNA strand. in Fig. 5 and the melting temperatures, $\mathrm{T}_{\mathrm{m}}$, are presented in Table 1. Attachment of a Tpy ligand at the N-end of the PNA duplexes did not significantly impact their melting temperature when compared to the melting temperature of the non-modified $\mathrm{P}_{\mathrm{N}}$ duplex (Table 1). This is in contrast to the effect of the $(S) \gamma$-methyl Tpy PNA monomer, which caused an increase in the melting temperature of ${ }^{\gamma} \boldsymbol{T p y y}_{\mathrm{N}_{\mathrm{N}}}{ }^{[19]} \mathrm{We}$ attribute this difference in the effect of Tpy on $\mathrm{T}_{\mathrm{m}}$ to the fact that when included in a PNA monomer, the Tpy can $\pi$-stack more effectively with the adjacent nucleobases and act as an overhang. Due to the shorter linkage between Tpy and PNA backbone when [2,2':6',2"-terpyridine]-4'-carboxylic acid is used to attach Tpy to the duplex, $\pi$-stacking is not possible or it is very weak; hence, the Tpy cannot stabilize the duplex.

Addition of 1 equivalent of $\mathrm{Cu}^{2+}$ leaves the $\mathrm{T}_{\mathrm{m}}$ of the ${ }^{G}$ Tpy $\mathrm{P}_{\mathrm{N}}$ duplex unchanged (Fig. $5 \mathrm{c}$ ), but causes a decrease of $\mathrm{T}_{\mathrm{m}}$ by $3{ }^{\circ} \mathrm{C}$ for duplexes ${ }^{A, l y s} \boldsymbol{T} \boldsymbol{T} \boldsymbol{y} \mathrm{P}_{\mathrm{N}}$ and ${ }^{\text {G,lys }} \boldsymbol{T} \boldsymbol{T} \boldsymbol{m} \boldsymbol{y} \mathrm{P}_{\mathrm{N}}$ (Fig. 5a and b). Thus, $\mathrm{Cu}^{2+}$ causes a slight decrease of the $\mathrm{T}_{\mathrm{m}}$ of duplexes that contain L-lysine only if a Tpy is situated across the L-lysine.

Addition of 0.5 or 1 equivalents of $\mathrm{Cu}^{2+}$ to all three duplexes ${ }^{G, l y s} \boldsymbol{T} \boldsymbol{p y} \mathrm{P}_{\mathrm{N}},{ }^{G} \boldsymbol{T} \boldsymbol{p} \boldsymbol{y} \mathrm{P}_{\mathrm{N}}$ and ${ }^{A, l y s} \boldsymbol{T} \boldsymbol{p y} \mathrm{P}_{\mathrm{N}}$ leads to a decrease in hyperchromicity (Fig. 5a-c) that suggests an enhancement in the efficiency of nucleobase $\pi$-stacking. This decrease in percent hyperchromicity together with the slight decrease in $\mathrm{T}_{\mathrm{m}}$ suggests that $\left[\mathrm{Cu}(\mathrm{Tpy})_{\mathrm{n}}\right]^{2+}$ complex has a negative steric impact on the duplex, possibly by intererence with the $\pi$-stacking of the nucleobases.

\subsection{UV-Vis Titrations}

UV-Vis titrations of the Tpy-modified duplexes were carried out to determine the stoichiometry of $\mathrm{Cu}^{2+}$ binding to Tpy. The dominant feature of the UV spectra of all PNAs is the $\pi \rightarrow \pi^{*}$ transition of the nucleobases at $260 \mathrm{~nm}$. Tpy-modified PNAs show

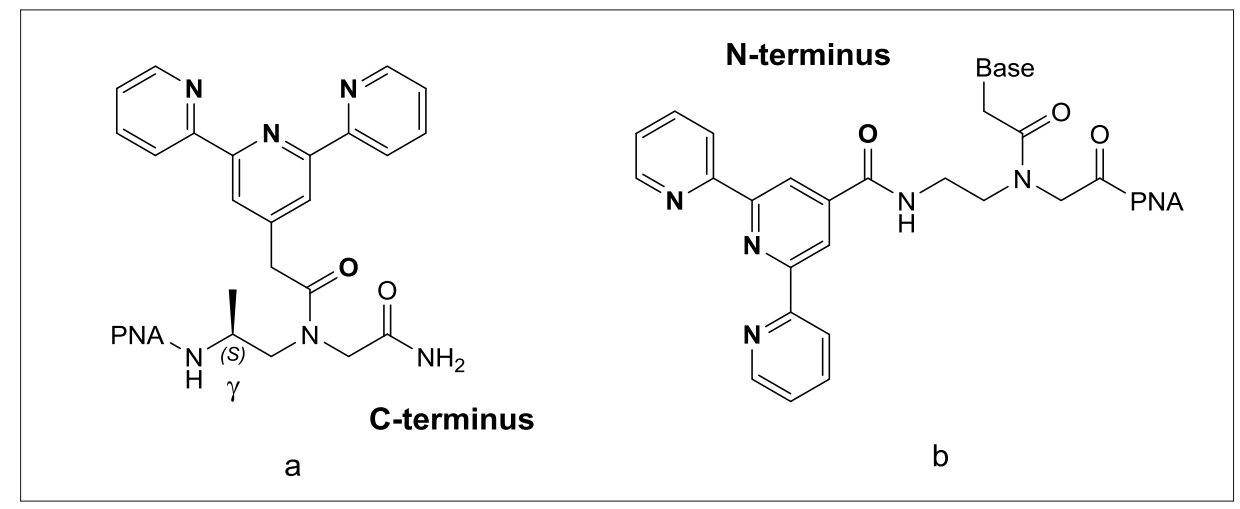

Fig. 4. The Tpy ligand can be introduced in PNA either by using a Tpy-containing PNA monomer as shown in (a), or by using a carboxylic acid of the Tpy as shown in (b). In the former case, the Tpy monomer can be placed at any position within a PNA strand; the figure shows the monomer placed at the C-end of a strand. In the latter case, Tpy can be attached only at the $\mathrm{N}$-end of a 
Table 1. Sequence, melting temperature $\mathrm{T}_{\mathrm{m}}$ and handedness of Tpy-PNA duplexes in the absence and presence of $\mathrm{Cu}^{2+a}$

\begin{tabular}{|c|c|c|c|c|c|c|}
\hline & Duplex & Strands & Sequences & No. $\mathrm{Cu}^{2+} \mathbf{E q}$. & $\mathbf{T}_{\mathrm{m}}\left[{ }^{\circ} \mathbf{C}\right]$ & Handedness \\
\hline \multirow{2}{*}{ 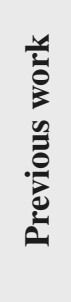 } & $P_{N}^{[11]}$ & & $\begin{array}{l}\mathrm{H}_{2} \mathrm{~N}^{-}{ }^{\mathrm{L} L y s-C A T C T A G T G A-H} \\
\quad \text { H-GTAGATCACT-LLys-NH}\end{array}$ & $\begin{array}{l}0 \\
1\end{array}$ & $\begin{array}{c}67 \\
67^{[19]}\end{array}$ & $\begin{array}{l}\text { Left } \\
\text { Left }\end{array}$ \\
\hline & ${ }^{\gamma} \boldsymbol{T} \boldsymbol{p} \boldsymbol{y} \mathrm{P}_{\mathrm{N}}{ }^{[19]}$ & & $\begin{array}{l}\mathrm{H}_{2} \mathrm{~N}-{ }^{\mathrm{L}} \text { Lys-CATCTAGTGA-H } \\
\text { H-GTAGATCACT- } \gamma \boldsymbol{T} \boldsymbol{p} \boldsymbol{y}-\mathrm{NH}_{2}\end{array}$ & $\begin{array}{l}0 \\
1\end{array}$ & $\begin{array}{l}72 \\
70\end{array}$ & $\begin{array}{l}\text { Right } \\
\text { Left }\end{array}$ \\
\hline \multirow{3}{*}{ 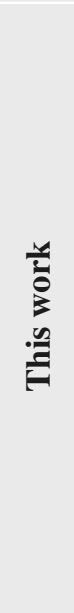 } & ${ }^{G, l y s} T p y \mathrm{P}_{\mathrm{N}}$ & P1P4 & $\begin{array}{l}\mathrm{H}_{2}{ }^{N-}-{ }^{-} L y s-C A T C T A G T G A-H \\
\\
\text { Tpy-GTAGATCACT- }{ }^{-} \text {Lys- } \mathrm{NH}_{2}\end{array}$ & $\begin{array}{c}0 \\
0.5 \\
1\end{array}$ & $\begin{array}{l}67 \\
66 \\
64\end{array}$ & $\begin{array}{l}\text { Right } \\
\text { Right } \\
\text { Left }\end{array}$ \\
\hline & ${ }^{A, l y s} \boldsymbol{T} \boldsymbol{p} \boldsymbol{y} \mathrm{P}_{\mathrm{N}}$ & P5P2 & $\begin{array}{l}\mathrm{H}_{2} \mathrm{~N}^{-}{ }^{\mathrm{L}} \text { Lys-CATCTAGTGA-Tpy } \\
\text { H-GTAGATCACT-L Lys-NH }\end{array}$ & $\begin{array}{c}0 \\
0.5 \\
1\end{array}$ & $\begin{array}{l}66 \\
65 \\
63\end{array}$ & $\begin{array}{c}\text { No CD } \\
\text { Left } \\
\text { Left }\end{array}$ \\
\hline & ${ }^{G} \boldsymbol{T p y} \mathrm{P}_{\mathrm{N}}$ & P3P4 & $\begin{array}{l}\mathrm{H}_{2} \mathrm{~N}-\mathrm{CATCTAGTGA-H} \\
\text { Tpy-GTAGATCACT-LLys-NH }\end{array}$ & $\begin{array}{c}0 \\
0.5 \\
1\end{array}$ & $\begin{array}{l}66 \\
65 \\
65\end{array}$ & $\begin{array}{l}\text { Right } \\
\text { Right } \\
\text { Right }\end{array}$ \\
\hline
\end{tabular}

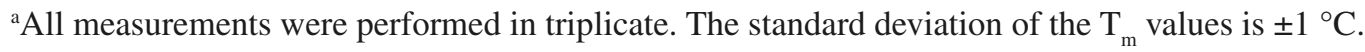

also a shoulder at $340 \mathrm{~nm}$, which is due to the $\pi \rightarrow \pi^{*}$ transitions of the Tpy ligand. The shoulder is shifted bathochromically to $355 \mathrm{~nm}$ in the presence of $\mathrm{Cu}^{2+}$ (Fig. 6a).

The titration curves of the three duplexes plotted at $351 \mathrm{~nm}$ are shown in Fig. 6b. All three duplexes show a relatively sharp inflection point at a 1:1 ratio of $\mathrm{Cu}^{2+}$ per PNA duplex. One isosbestic point was observed at $325 \mathrm{~nm}$. These results suggest the formation of a mono-terpyridine $[\mathrm{Cu}(\mathrm{Tpy})]^{2+}$ complex between the Tpy-containing PNA duplexes and $\mathrm{Cu}^{2+}$. The strong Jahn-Teller effect of $\mathrm{Cu}^{2+}$ ions, the presence of coordinating $\mathrm{HPO}_{4}^{2-}$ and $\mathrm{H}_{2} \mathrm{PO}_{4}^{-}$ions in the buffer, and of L-lysine in ${ }^{4}, l y s \boldsymbol{T} \boldsymbol{T} \boldsymbol{y} \mathrm{P}_{\mathrm{N}}$ and ${ }^{A, l y s}$ T $\boldsymbol{T y} \mathrm{P}_{\mathrm{N}}$, as well as the steric effect of the PNA duplexes on metal complexes may be the reasons that preclude the formation of a $\left[\mathrm{Cu}(\mathrm{Tpy})_{2}\right]^{2+}$ complex. Nonetheless, it is also possible that the inflection point at the $\mathrm{Cu}^{2+}$ to duplex PNA ratio of 0.5 is not sharp enough to be observed in the UV-Vis titration curve.

\subsection{Spectroscopy}

CD spectroscopy was employed to study the effect of terminal Tpy moiety on the chiral induction effect of L-lysine, and to monitor the outcome of $\mathrm{Cu}^{2+}$ coordination to the Tpy on the duplex handedness.

Duplex ${ }^{\text {G,lys }}$ Tpy $\mathrm{P}_{\mathrm{N}}$ annealed in the absence of $\mathrm{Cu}^{2+}$ ions shows a positive Cotton effect at 220 and $256 \mathrm{~nm}$, which is indicative of a right-handed P-helix (Fig. 7a). Interestingly, annealing of the same duplex in the presence of 0.5 equivalents of

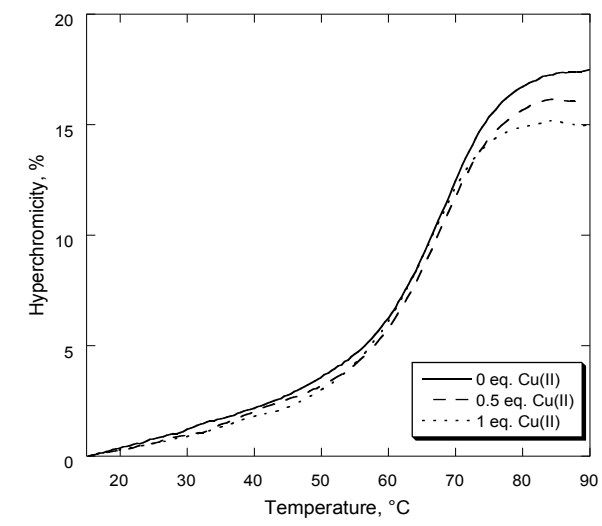

a
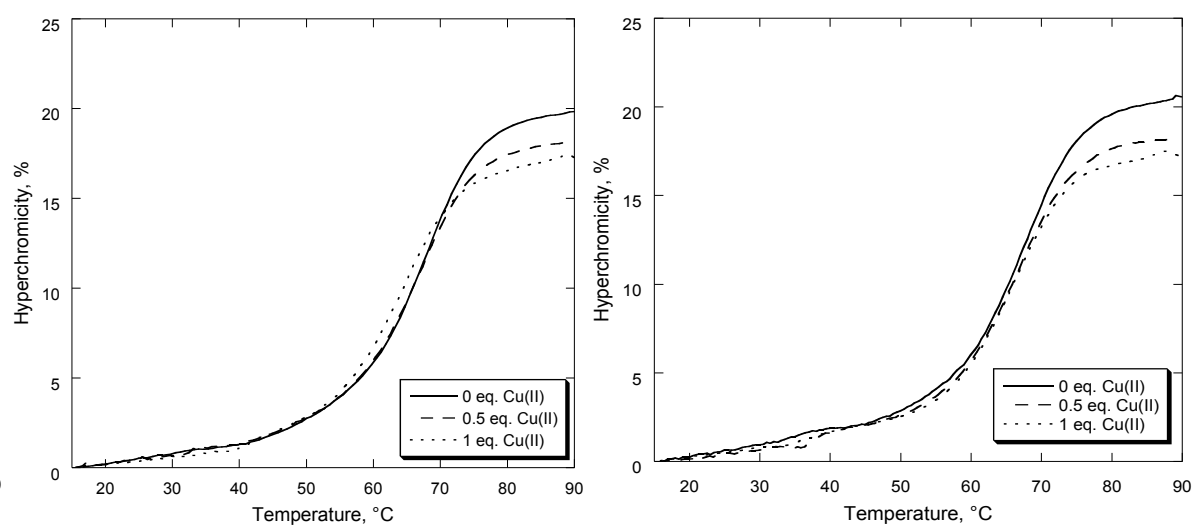

b

$\mathrm{C}$

Fig. 5. Melting curves of (a) ${ }^{A, l y s} T_{p y P} P_{N}$, (b) ${ }^{G, l y s} T_{p y} P_{N}$, and (c) ${ }^{G} T_{p y P} P_{N}$ annealed in the absence (solid lines) and presence of 0.5 or 1 equivalents of $C u^{2+}$ (dashed lines). Solutions are $10 \mu \mathrm{M}$ PNA duplex in $\mathrm{pH} 710 \mathrm{mM}$ sodium phosphate buffer. 

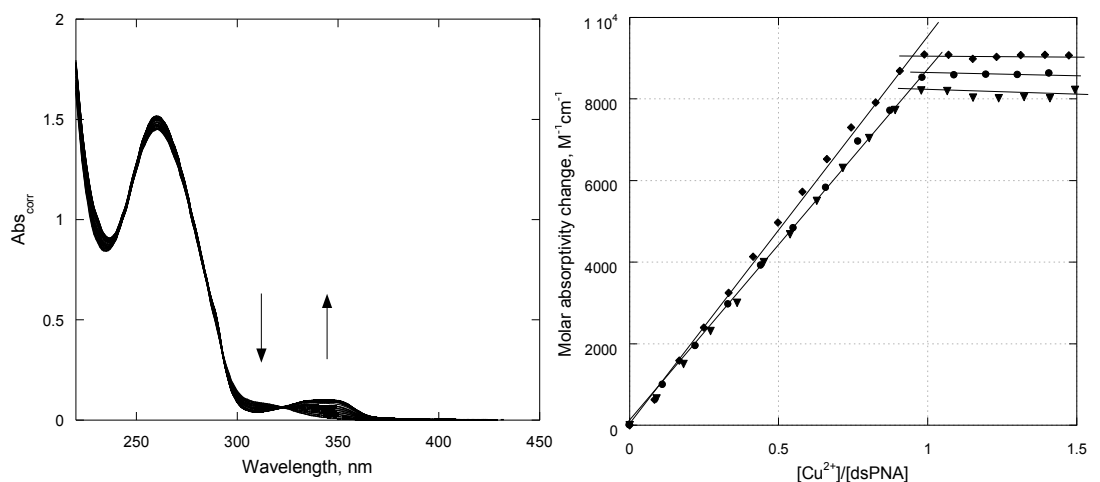

Fig. 6. Spectrophotometric titration of (a) ${ }^{\text {G,lys }} \operatorname{Tpy}_{\mathrm{N}}$ and (b) the superimposed titration curves of

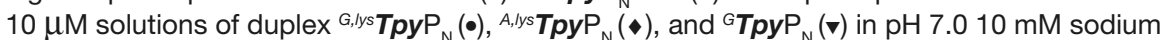
phosphate buffer with $500 \mu \mathrm{M}$ aqueous solution of $\mathrm{CuCl}_{2}, \mathrm{~T}=25^{\circ} \mathrm{C}$.

$\mathrm{Cu}^{2+}$ lead to an increase in the intensity of the CD signals at both 220 and $256 \mathrm{~nm}$, which suggests an enhancement in the cooperativity of the chiral induction. This increase in CD intensity and the increase in hyperchromicity observed on the thermal annealing curve hint at the formation of $\left[\mathrm{Cu}(\mathrm{Tpy})_{2}\right]^{2+}$ or another intermediate, which is not detectable by UV-Vis titration. However, when the same two PNA oligomers were annealed in the presence of 1 equivalent of $\mathrm{Cu}^{2+}$ the duplex adopted a left-handed structure.

Surprisingly, duplex ${ }^{A, \text { lys }} \boldsymbol{T p y y}_{\mathrm{N}}$ annealed in the absence of $\mathrm{Cu}^{2+}$ ions showed no CD features (Fig. 7b). Hence, the double-stranded PNA was either achiral or the solution contained a racemic mixture of duplexes with opposite handedness. In the presence of 0.5 or 1 equivalents of $\mathrm{Cu}^{2+}$, the duplex ${ }^{A, l y s} \boldsymbol{T} \boldsymbol{p y} \mathrm{P}_{\mathrm{N}}$ was left-handed, as shown by the negative exciton coupling patterns at 220 and $256 \mathrm{~nm}$.

The ${ }^{G} \boldsymbol{T} \boldsymbol{p y} \mathrm{P}_{\mathrm{N}}$ duplex, which lacks a lysine residue opposite to the Tpy moiety showed intense positive CD at $260 \mathrm{~nm}$, which are indicative of a right-handed structure in the absence and in the presence of $\mathrm{Cu}^{2+}$ ions (Fig. 7c). Additionally, in the presence of $\mathrm{Cu}^{2+}$, one observes the positive $\mathrm{CD}$ signals at $340 \mathrm{~nm}$, which suggest the formation of a chiral Tpy-containing complex.

\section{Discussions}

Several different studies described the chiral induction effect of a terminal chiral group incorporated in PNA. ${ }^{20]}$ Wittung et al. determined that the L-amino acids with hydrophobic side chains stabilize left-handed helices, whereas amino acids with hydrophilic side chains stabilize right-handed helices. ${ }^{[13]}$ Attachment of large aromatic moieties at the $\mathrm{N}$-ends of L-lysine-containing PNA has been studied. Porphyrins have been introduced in parallel PNA duplexes ${ }^{[21]}$ (duplexes in which the complementary strands have their C-ends opposite to each other), and fluo- rescein and rhodamine dyes in antiparallel PNA duplexes.[22] The CD spectra of the PNA duplex containing one N-terminal porphyrin showed no major conformational changes with respect to the nonmodified duplex. However, bis-porphyrin/ PNA conjugates partially lost their helicity, which was attributed to the steric interactions resulting from the cofacial arrangement of the two aromatic entities. ${ }^{[21]}$ In a study by Ratilainen et al. it was concluded that the equilibrium between left- and right-handed PNA duplexes is strongly affected by the attachment of fluorescein to the N-end via a flexible linker; a reversal of the sense of handedness of PNA duplex was observed.[22]

In the present study, we examined PNA duplexes that contain in complementary position to a terminal L-lysine the aromatic metal-binding ligand Tpy (soldier) which in the absence of $\mathrm{Cu}^{2+}$ could form cation- $\pi$ interactions with the $\mathrm{NH}_{3}^{+}$of the L-lysine. This interaction would interfere with the chiral induction effect of the $\mathrm{C}$-terminal $\mathrm{L}$ lysine (sergeant). In the presence of $\mathrm{Cu}^{2+}$ ions, Tpy can form $\left[\mathrm{Cu}(\mathrm{Tpy})_{\mathrm{n}}\right]^{2+}$ complexes; in turn, the metal coordination to Tpy could eliminate the Tpy interaction with lysine, and the amino acid would exert its typical chiral induction effect that leads to a left-handed duplex.

Indeed, in both duplexes that contain a Tpy complementary to an L-lysine, namely ${ }^{G, l y s} \boldsymbol{T}$ py $\mathrm{P}_{\mathrm{N}}$ and ${ }^{A, l y s} \boldsymbol{T} \boldsymbol{T} \boldsymbol{y} \mathrm{P}_{\mathrm{N}}$, the $\mathrm{CD}$ signal does not show the exciton coupling pattern indicative of a left-handed duplex observed for $\mathrm{P}_{\mathrm{N}}$ (Fig. 7). These observations support the idea that an interaction between the L-lysine and Tpy prevents the amino acid from relaying its left-handedness to the PNA duplex. $\mathrm{Cu}^{2+}$ addition to the duplexes ${ }^{\text {G,lys }} \boldsymbol{T} \boldsymbol{\text { py }} \mathrm{P}_{\mathrm{N}}$ and ${ }^{A, l y s} \boldsymbol{T} \boldsymbol{T} \boldsymbol{y} \mathrm{P}_{\mathrm{N}}$ leads to formation of $[\mathrm{Cu}(\mathrm{Tpy})]^{2+}$ and causes the

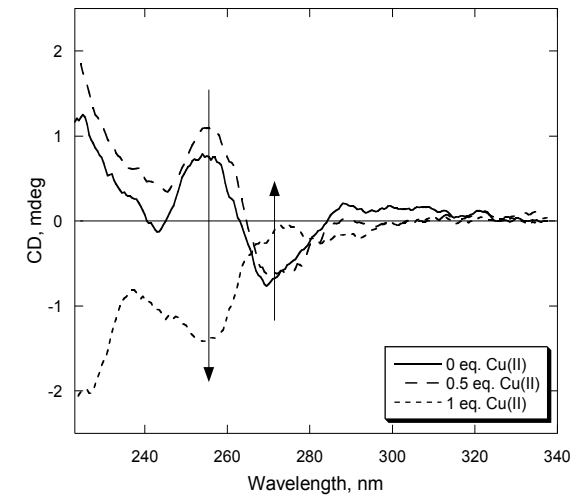

a
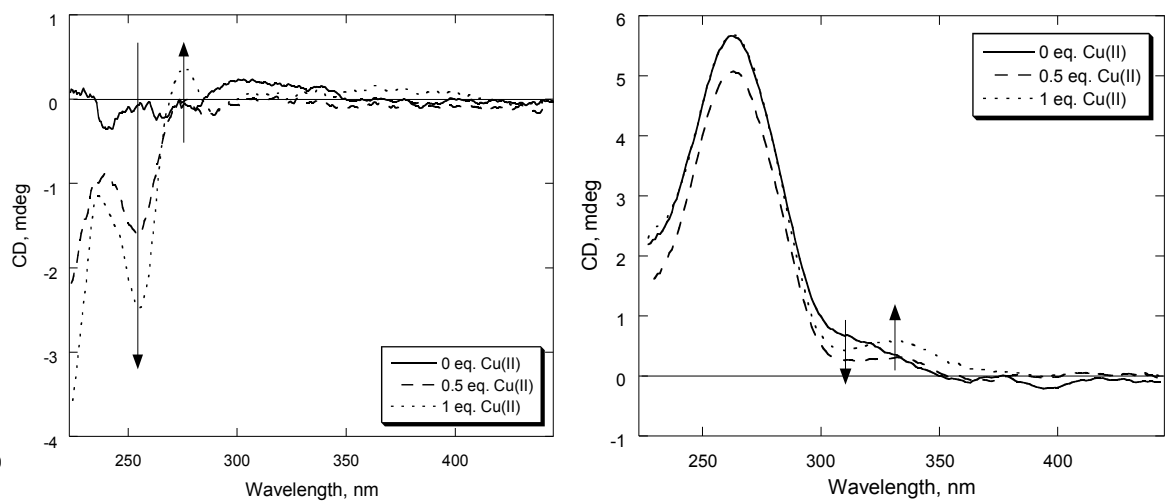

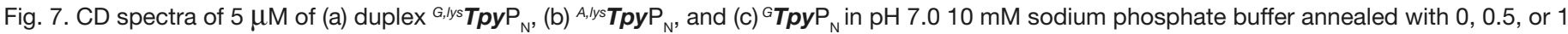
equivalents of $\mathrm{Cu}^{2+}$. 
observation of a $\mathrm{CD}$ spectrum indicative of left-handedness (Fig. 7), supporting the idea that L-lysine can now exert its known chiral induction effect. While we speculated that a cation- $\pi$-interaction between the $\mathrm{NH}_{3}^{+}$group of the L-lysine and Tpy may be underlying the effect of Tpy on the chiral induction effect of L-lysine, it is also possible that Tpy affects the duplex itself in a manner dependent on the presence or absence of $\mathrm{Cu}^{2+}$. In terms of the 'sergeantand-soldiers' effect, N-terminal Tpy may be regarded as a disobeying soldier who does not follow the sergeant's orders.

The lack of additional stabilization of the Tpy-modified duplexes used in this study, i.e. no overhang effect that was previously observed for the $(S) \gamma$-Tpy monomer, ${ }^{[19]}$ suggests that there is no effective stacking between Tpy and neighboring nucleobases. This lack of stabilization may be due to the relatively short connection between Tpy and the last $\mathrm{N}$-terminal monomer that does not allow for an optimum arrangement for Tpy to $\pi$-stack.

The CD spectrum of the ${ }^{G} \boldsymbol{T} \boldsymbol{T} \boldsymbol{y} \mathrm{P}_{\mathrm{N}}$ duplex, which contains a Tpy not complementary to the L-Lys but at the opposite end of the duplex, shows a broad positive feature at $260 \mathrm{~nm}$ suggestive of a right-handed structure, but different from the spectrum of a P-helix. This observation supports the idea that the effect of the Tpy on the chiral induction effect of the L-Lys is determined by the ligand interaction with the duplex itself rather than by its interaction with $\mathrm{L}$ Lys. The Tpy ligand interaction with the duplex is unlikely to be by $\pi$-stacking at the end of the duplex as it was established for the ${ }^{\gamma} \boldsymbol{T} \boldsymbol{p y} \mathrm{P}_{\mathrm{N}}$ duplex because the linker used in the current study is significantly shorter and less flexible than that used to attach Tpy to ${ }^{\gamma} \boldsymbol{T} \boldsymbol{p} \boldsymbol{P} \mathrm{P}_{\mathrm{N}}$. This inference is supported by the fact that the $\mathrm{T}_{\mathrm{m}}$ of the duplex increased for ${ }^{\gamma} \boldsymbol{T} \boldsymbol{p} \boldsymbol{y} \mathrm{P}_{\mathrm{N}}$ but not for the duplexes reported here.

In summary, we have found that the chiral induction effect of a chiral 'sergeant' can be modulated using achiral 'soldiers' in the case of PNA. A systematic experimental and computation study is underway in our laboratory to further the understanding of the modulation effect exerted by Tpy on the chiral induction effect of L-Lys and its potential applications to supramolecular structures and nanodevices.

\section{Conclusions}

Investigations carried out on three PNA duplexes that contained C-terminal L-lysine (sergeant) and N-terminal Tpy (soldier) have shown that the chiral induction effect of the amino acid can be modulated by an achiral metal-binding ligand. This effect is different from that observed by Bezer et al. in which the helical sense induction effect of the chiral monomer itself was turned on/ off by means of metal coordination. ${ }^{[19]}$ Our findings may be of value in the design of hybrid inorganic-nucleic acid structures with tunable helicity and helical sense for use in chiroptical nanodevices.

\section{Materials and Methods}

\subsection{Solid-phase PNA Synthesis}

[2,2':6',2'-terpyridine]-4'-carboxylic acid was purchased from Alfa Aesar and used without further purification. The PNA oligomers were prepared by solidphase peptide synthesis on an MBHA resin (Peptides International) downloaded with L-lysine to an 0.1 meq./g NH Boc/Z-protected PNA monomers were purchased from PolyOrg and used without further purification. The PNA oligomers were cleaved from the solid support using a mixture of $m$-cresol/thioanisole/TFMSA/ TFA (1:1:2:6) for $1 \mathrm{~h}$. Cleaved PNA was precipitated using diethyl ether and purified by reversed-phase HPLC using a C18 silica column on a Waters 600. Absorbance was measured with a Waters 2996 photodiode array detector. Characterization of the oligomers was done by MALDI-ToF mass spectrometry using $\alpha$-cyano-4hydroxycinnamic acid matrix (10 mg/ $\mathrm{mL}$ in 1:1 water/acetonitrile, $0.1 \% \mathrm{TFA}$ ). An Applied Biosystems Voyager workstation with delayed extraction was used for MALDI-ToF mass spectrometry. The calculated and experimental values of $\mathrm{m} / \mathrm{z}$ for $\left[\mathrm{PNA}+\mathrm{H}^{+}\right]$species are provided in Table 2 .

\subsection{UV-Vis Spectroscopy}

UV-Vis experiments were performed on a Varian Cary 3 spectrophotometer equipped with a programmable temperature block, in quartz cells of 10 -mm optical path. PNA stock solutions were prepared in nanopure water $(18.2 \mathrm{M} \Omega \cdot \mathrm{cm})$. PNA concentrations were determined by UV-Vis spectrophotometry, assuming $\varepsilon(260 \mathrm{~nm})$ $=8600,6600,13700$, and $11700 \mathrm{M}^{-1} \cdot \mathrm{cm}^{-1}$ for the T, C, A, and $\mathrm{G}$ monomers, and $\varepsilon(260$ $\mathrm{nm})=9750 \mathrm{M}^{-1} \cdot \mathrm{cm}^{-1}$ for the Tpy moiety. ${ }_{[}{ }^{19]}$ PNA solutions for melting curves and titration had concentrations in the 5-10 micromolar range, and were prepared in $\mathrm{pH} 7.0$ $10 \mathrm{mM}$ sodium phosphate buffer.

UV melting curves were recorded in the temperature range $15-90{ }^{\circ} \mathrm{C}$ for both cooling and heating modes, at the rate of $1{ }^{\circ} \mathrm{C} / \mathrm{min}$. The melting curves were measured at the maximum absorbance of PNA, which is $260 \mathrm{~nm}$. Prior to the measurement of the melting profiles, the solutions were kept at $90{ }^{\circ} \mathrm{C}$ for $10 \mathrm{~min}$. $\mathrm{T}_{\mathrm{m}}$ was taken at the inflection point of the Boltzmann sigmoidal fit function (Eqn. (1)) that assumes a two-state model:

$$
M_{1}+\frac{M_{2}}{1+\exp \left(\frac{M_{3}-M_{0}}{M_{4}}\right)}
$$

where $M_{1}=0.1 ; M_{2}=17 ; M_{3}=71 ; M_{4}=5$.

UV-Vis titrations were carried out by addition of aliquots of standard $500 \mu \mathrm{M}$ $\mathrm{CuCl}_{2}$ aqueous solution to the PNA solutions in $\mathrm{pH}$ 7.0, $10 \mathrm{mM}$ sodium phosphate buffer. The absorbance at each wavelength $\lambda, A_{\lambda}$, was corrected $\left(A_{\lambda, \text { corr }}\right)$ for dilution using Eqn. (2):

$$
A_{\lambda, \text { corr }}=\frac{V_{\text {initial }}}{V_{\text {initial }}+V_{\text {added }}} \cdot A_{\lambda}
$$

where $A_{\lambda}$ stands for the measured absorbance at wavelength $\lambda, V_{\text {initial }}$ represents the initial volume of solution in the cuvette (1 $\mathrm{mL}$ ), and $V_{\text {added }}$ represents the total volume of the $\mathrm{CuCl}_{2}$ solution in the cuvette after each aliquot addition.

\subsection{Spectroscopy}

CD spectra were measured for $5 \mu \mathrm{M}$ PNA solutions in $10 \mathrm{mM}$ sodium phosphate buffer ( $\mathrm{pH} 7.0)$ on a JASCO J-715 spectropolarimeter equipped with a thermoelectrically-controlled single cell holder. CD spectra were collected at $20{ }^{\circ} \mathrm{C}$, using $1 \mathrm{~nm}$ bandwidth, $1 \mathrm{~s}$ response time, $50 \mathrm{~nm} / \mathrm{min}$ speed, $20 \mathrm{mdeg}$ sensitivity, and 10 scan accumulation.

\begin{tabular}{|c|c|c|c|}
\hline Name & PNA sequence & Calcd. $\mathbf{m} / \mathbf{z}$ & Found $\mathbf{m} / \mathbf{z}$ \\
\hline P1 & $\mathrm{H}_{2} \mathrm{~N}-\mathrm{L} L y s-C A T C T A G T G A-H$ & 2877.83 & 2878.62 \\
\hline $\mathrm{P} 2$ & $\mathrm{H}_{2} \mathrm{~N}-{ }^{\mathrm{L} L y s}-\mathrm{TCACTAGATG}-\mathrm{H}$ & 2877.83 & 2876.64 \\
\hline P3 & $\mathrm{H}_{2} \mathrm{~N}$-CATCTAGTGA-H & 2749.65 & 2747.10 \\
\hline P4 & $\mathrm{H}_{2} \mathrm{~N}-{ }^{\mathrm{L} L y s-T C A C T A G A T G-T p y}$ & 3137.10 & 3134.77 \\
\hline P5 & $\mathrm{H}_{2} \mathrm{~N}-{ }^{\mathrm{L}}$ Lys-CATCTAGTGA-Tpy & 3137.10 & 3137.02 \\
\hline
\end{tabular}

Table 2. Molecular weight and MALDI MS data for the PNA oligomers 


\section{Acknowledgements}

We thank the U.S. National Science Foundation for support of this work (CHE1310441). The Center for Molecular Analysis at Carnegie Mellon University was supported in part by the NSF (CHE-9808188 and DBI9729351).

Received: April 15, 2018

[1] J. W. Canary, Z. Dai, S. Mortezaei, in 'Comprehensive Chirality', Vol. 8, Elsevier B.V., 2012, pp. 600, DOI: 10.1016/B978-0-08095167-6.00850-8.

[2] Y. Kim, B. Yeom, O. Arteaga, S. J. Yoo, S. G. Lee, J. G. Kim, N. A. Kotov, Nat. Mater. 2016, 15, 461, DOI: $10.1038 /$ nmat4525.

[3] B. L. Feringa, R. A. Van Delden, B. L. Feringa, R. a Van Delden, N. Koumura, N. Koumura, E. M. Geertsema, E. M. Geertsema, Chem. Rev. 2000, 100, 1789, DOI: 10.1021/cr9900228.

[4] V. Varade, T. Z. Markus, K. Vankayala, N. Friedman, M. Sheves, D. H. Waldeck, R. Naaman, Phys. Chem. Chem. Phys. 2017, 20, 1091, DOI: 10.1039/C7CP06771B.
[5] E. Yashima, N. Ousaka, D. Taura, K. Shimomura, T. Ikai, K. Maeda, Chem. Rev. 2016, 116, 13752, DOI: 10.1021/acs.chemrev.6b00354.

[6] L. J. Prins, P. Timmerman, D. N. Reinhoudt, J. Am. Chem. Soc. 2001, 123, 10153, DOI: 10.1021/ja010610e.

[7] D. B. Amabilino, in 'Chirality Supramol. Assem.', John Wiley \& Sons Ltd., 2017, pp. 159, DOI: $10.1002 / 9781118867334 . c h 6$.

[8] R. Naaman, D. H. Waldeck, J. Phys. Chem. Lett. 2012, 3, 2178, DOI: 10.1021/jz300793y.

[9] L.-J. Chen, H.-B. Yang, M. Shionoya, Chem. Soc. Rev. 2017, 46, 2555, DOI: 10.1039/ $\mathrm{C} 7 \mathrm{CS} 00173 \mathrm{H}$

10] M. Balaz, B. C. Li, J. D. Steinkruger, G. A. Ellestad, K. Nakanishi, N. Berova, Org. Biomol. Chem. 2006, 4, 1865, DOI: 10.1039/b603409h.

[11] P. Wittung, P. E. Nielsen, O. Buchardt, M Egholm, B. Nordén, Nature 1994, 368, 561, DOI: $10.1038 / 368561 \mathrm{a} 0$.

[12] H. Rasmussen, S. J. Kastrup, J. N. Nielsen, J. M. Nielsen, P. E. Nielsen, Nat. Struct. Biol. 1997, 4, 98, DOI: 10.1038/nsb0297-98.

[13] P. Wittung, M. Eriksson, R. Lyng, P. E. Nielsen, B. Nordén, J. Am. Chem. Soc. 1995, 117, 10167.

[14] A. Otto, A. Porzel, J. Schmidt, W. Brandt, L. Wessjohann, F. Totsingan, V. Jain, W. C. Bracken, A. Faccini, T. Tedeschi, R. Marchelli, R. Corradini, N. R. Kallenbach, M. M. Green,
Macromolecules 2010, 43, 2692, DOI: 10.1021/ ma902797f.

[15] H. Rasmussen, T. Liljefors, B. Petersson, P. E. Nielsen, J. S. Kastrup, J. Biomol. Struct. Dyn. 2004, 21, 495, DOI: 10.1080/07391102.2004.10506943.

[16] B. Petersson, B. B. Nielsen, H. Rasmussen, I. K. Larsen, M. Gajhede, P. E. Nielsen, J. S. Kastrup, J. Am. Chem. Soc. 2005, 127, 1424, DOI: $10.1021 / \mathrm{ja} 0458726$.

[17] J. I. Yeh, E. Pohl, D. Truan, W. He, G. M. Sheldrick, S. Du, C. Achim, Chem. - A Eur. J. 2010, 16, 11867, DOI: 10.1002/chem. 201000392.

[18] A. Dragulescu-Andrasi, S. Rapireddy, B. M. Frezza, C. Gayathri, R. R. Gil, D. H. Ly, J. Am. Chem. Soc. 2006, 128, 10258, DOI: 10.1021/ ja0625576.

[19] S. Bezer, S. Rapireddy, Y. A. Skorik, D. H Ly, C. Achim, Inorg. Chem. 2011, 50, 1, DOI: 10.1021/ic200855p.

[20] F. Totsingan, V. Jain, M. M. Green, Artif. DNA. PNA XNA 2012, 3, 31.

[21] A. Accetta, A. G. Petrovic, R. Marchelli, N. Berova, R. Corradini, Chirality 2015, 27, 864 , DOI: 10.1002/chir.22521.

[22] T. Ratilainen, A. Holmén, E. Tuite, G. Haaima, L. Christensen, P. E. Nielsen, B. Nordén, Biochemistry 1998, 37, 12331, DOI: 10.1021/ bi9808722. 